\title{
Therapeutic Effects of Momordica charantia L. Ethanolic Extract on Acetic Acid-Induced Ulcerative Colitis in Rats
}

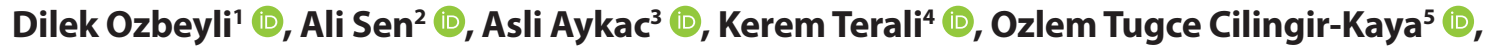 \\ Ismail Senkardes ${ }^{6}$ iD, Goksel Sener ${ }^{7}$
}

\author{
'Marmara University, Vocational School of Health Services, Department of Pathology, Laboratory Techniques, Istanbul, Turkey \\ 2Marmara University, Faculty of Pharmacy, Department of Pharmacognosy, Istanbul, Turkey \\ ${ }^{3}$ Near East University, Department of Biophysics, Northern Cyprus KKTC \\ ${ }^{4}$ Girne American University, Faculty of Medicine, Department of Medical Biochemistry, Northern Cyprus KKTC \\ 5Marmara University, School of Medicine, Department of Histology and Embryology, Istanbul, Turkey \\ ${ }^{6}$ Marmara University, Faculty of Pharmacy, Department of Pharmaceutical Botany, Istanbul, Turkey \\ ${ }^{7}$ Fenerbahce University, Vocational School of Health Services, Istanbul, Turkey
}

ORCID IDs of the authors: D.O. 0000-0002-4141-6913; A.S. 0000-0002-2144-5741; A.A. 0000-0002-4885-5070; K.T. 0000-0002-9964-6383; O.T.C.K. 0000-0002-2591-9174; I.S. 0000-0002-2656-0319; G.S. 0000-0001-7444-6193

Please cite this article as: Ozbeyli D, Sen A, Aykac A, Terali K, Cilingir-Kaya OT, Senkardes I, Sener G. Therapeutic Effects of Momordica charantia L. Ethanolic Extract on Acetic Acid-Induced Ulcerative Colitis in Rats. Eur J Biol 2021; 80(2): 119-128. DOI: 10.26650/EurJBiol.2021.1016222

\section{ABSTRACT}

Objective: This study aims to investigate the effect of Momordica charantia L. (MoC) ethanolic extract on ulcerative colitis (UC) and was explored in vitro and in vivo.

Materials and Methods: The rats were divided into control (C), saline-treated colitis (AA), MoC extract-treated colitis $(\mathrm{AA}+\mathrm{MoC})$, and sulfasalazine (SS)-treated colitis (AA+SS) groups. Colitis was induced by acetic acid. MoC extract, SS or saline were given to the related groups for 3 days. Interleukine-1 $\beta$, malondialdehyde, glutathione levels, myeloperoxidase activity, bax/bcl-2 ratio, caspase- 9 and caspase- 3 levels were measured in colon and macroscopic and histopathologic examinations were done. Total phenolic/flavonoid content and biological activity of MoC were evaluated by in vitro analysis.

Results: Compared to the control group, with acetic acid application interleukin-1 $\beta$ levels, myeloperoxidase activity, malondialdehyde levels, bax/bcl-2 ratio, caspase- 9 and caspase-3 levels were significantly upregulated, while glutathione levels were significantly decreased in the AA group. In contrast, $\mathrm{MoC}$ and SS treatments reduced interleukin- $1 \beta$, malondialdehyde levels, myeloperoxidase activity, bax/bcl-2 ratio, and caspase- 9 and caspase-3 levels. Glutathione levels increased upon MoC or SS treatment. Increased macroscopic and microscopic scoring with AA improved with MoC or SS treatment, but the MoC or SS treated groups had higher score values than the control. Also, in vitro results showed that MoC exhibited 2,2-diphenyl-1picrylhydrazyl and 2,2'-azino-bis-3-ethylbenzothiazoline-6-sulfonic acid radical scavenging activity as well as significant antilipoxygenase activity. In addition, MoC extract showed a potent anti-inflammatory activity compared to standard indomethacin.

Conclusion: Our biochemical, in vitro and histopathologic analysis indicate that MoC is likely to prove beneficial in UC therapy. Keywords: Momordica charantia L., ulcerative colitis, apoptosis, oxidative stress, radical scavenging activity, anti-lipoxygenase activity

\section{INTRODUCTION}

Ulcerative colitis (UC) affects millions of people worldwide and is characterized by recurrent mucosal inflammation and ulceration of the large intestine (1).
Although the exact aetiology of UC is not well documented, it involves a dysregulated immune system as well as environmental effects. Current UC treatments are based on the suppression of inflammation (2). The 
acetic acid (AA)-induced UC model represents a good model for clinical, biochemical, and histological examinations in coIonic inflammation (3). AA causes major epithelial damage and is characterized by increased leukocyte infiltration, increased inflammatory mediators, vascular dilatation, oedema, and large, massive ulceration of the non-transmural large intestine (4). Several cytokines are involved in the pathogenesis of UC, including tumour necrosis factor (TNF)- $\alpha$ and interleukin $1 \beta$ (IL$1 \beta)$ (5). Oxidative stress plays a significant role in the aetiology of UC in such a way that reactive oxygen species (ROS) formation during inflammation causes apoptosis-mediated cellular death and loss of epithelial cells. However, an increase in the apoptotic cells leads to a deterioration of the epithelial defence in the colon and accelerates mucosal inflammation (6).

Current therapies mainly aim at decreasing inflammation using anti-inflammatory drugs as well as at reducing oxidative stress (3). Aminosalicylates, corticosteroids, and immunosuppressants that are used in the treatment of mild and moderate UC provide regression of disease symptoms. One of them, sulfasalazine (SS), which is utilized as standard therapy in the disease, corresponds to an aminosalicylate derivative. The 5-aminosalicylic acid component of SS is liable for its therapeutic effect, and the majority of its side effects are linked to the sulphapyridine portion (5). However, treatments using these agents have drug-related side effects that can lead to some problems (7). On the other hand, natural plants have long been used to treat various diseases and to avoid the side effects of drugs. Momordica charantia L. (MoC), also known as bitter gourd, bitter apple, or bitter melon, is a green to yellow-coloured flowering plant and a member of the Cucurbitaceae family. MoC widely grows in Asia and the Mediterranean area, and the main phytochemical ingredients of $\mathrm{MoC}$ include essentially flavonoids, phenolic acids, cucurbitane-type triterpenoid saponins (momordicine, momordin, and momordicoside (8-10). Standard constituents of $\mathrm{MoC}$ are charantin (a mixture of 5.25-stigmasteryl glucoside and $\beta$-sitosteryl glucoside) and momordicine, which are steroidal saponins (811). Momordicine and charantin are mainly responsible for the beneficial activities and bitterness of MoC (8-12). MoC has been reported to have more biological effects such as antioxidant, anti-apoptotic and anti-inflammatory $(9,12,13)$. In addition, MoC fruit is used externally for the quick healing of abrasions and internally for the cure of peptic ulcers in Turkish folk medicine (14). MoC has been shown to alleviate dextran sulfate sodium (DSS)-induced colitis in mice by depressing inflammatory cytokines and increasing regulatory T cells (15). Additionally, $\mathrm{MoC}$ has been demonstrated to have significant anti-inflammatory effects on trinitrobenzene (TNBS)-induced colitis that mimics Crohn's disease by reducing myeloperoxidase (MPO) activity and cytokine levels in rats (10).

During colitis induction, administered MoC therapy can improve colonic damage ameliorated by decreased colonic oxidative stress and apoptosis. Therefore, the present study was designed to assess the potential antioxidant, anti-inflammatory and anti-apoptotic effects of $\mathrm{MoC}$ on AA-induced ulcerative rat models through in vitro and in vivo analysis.

\section{MATERIALS AND METHODS}

\section{Chemicals}

Acetic acid, dimethyl sulfoxide (DMSO), 2-(4-(2-hydroxyethyl) piperazin-1-yl) ethanesulfonic acid (HEPES), hexadecyltrimethylammonium bromide (HETAB), N-(2-Hydroxyethyl) piperazine-N'-(2-ethanesulfonic acid), trichloroacetic acid (TCA), thiobarbituric acid (TBA), 5,5'-dithiobis(2-nitrobenzoic acid) (DTNB), o-Dianisidine, $\mathrm{Na}_{2} \mathrm{HPO}_{4} .2 \mathrm{H}_{2} \mathrm{O}, \mathrm{K}_{2} \mathrm{HPO}_{4}, 2$,2-diphenyl-1-picrylhydrazyl (DPPH), 2,2-azino-bis-3-ethylbenzothiazoline-6-sulfonic acid (ABTS), potassium persulfate, indomethacine, ascorbic acid, trolox, lipoxidase from Glycine max (soybean), Folin-Ciocalteu reagent, linoleic acid, formaldehyde, dithiothreitol (DTT), glycerol, Tris- $\mathrm{HCl}$, Ethylenediaminetetraacetic acid (EDTA), and Triton $\mathrm{X}-100, \mathrm{NaNO}_{2}, \mathrm{AlCl}_{3} \cdot 6 \mathrm{H}_{2} \mathrm{O}, \mathrm{NaOH}$, methanol, and ethanol were supplied by Sigma (Sigma-Aldrich, St. Louis, MO, USA). All antibodies for immunoblotting were purchased from Santa Cruz Biotechnology Inc (Santa Cruz, CA, USA). Sodium pentobarbital was purchased from IE Ulagay (Istanbul, Turkey). All other chemicals were of the purest grade commercially available.

\section{MoC Extract Preparation}

MoC fruits were collected from the rural district of Gemlik in Bursa, Turkey in August 2019 and identified by Dr. İsmail Şenkardeş from the Department of Pharmaceutical Botany, Faculty of Pharmacy, Marmara University. The voucher specimens were deposited in the Herbarium Faculty of Pharmacy, Marmara University (Marmara No: 22446). Fresh fruit with seeds was blended and then were macerated by $95 \%$ ethanol $(1000 \mathrm{~mL})$ for $48 \mathrm{~h}$. The extraction process was repeated until the solvent became colourless. The filtrate was evaporated and concentrated at $40^{\circ} \mathrm{C}$. The obtained dried ethanol extract of MoC was stored at $+4^{\circ} \mathrm{C}$ until further analysis.

\section{In Vitro Antioxidant and Anti-inflammatory Activity of MoC}

The DPPH radical scavenging capacity measurement of MoC extract was performed in line with a previously reported procedure by Zou et al. (16). In short, $10 \mu \mathrm{L}$ of extract or standard ascorbic acid in DMSO at different concentrations were mixed with $190 \mu \mathrm{L}$ of $0.1 \mathrm{mM}$ DPPH solution in methanol in a 96-well plate. Mixtures were left to incubate for $30 \mathrm{~min}$ in the dark at room temperature. Then, the absorbance was taken at $517 \mathrm{~nm}$. Tests were carried out in triplicate.

The radical scavenging activity percentage of extracts and compounds against DPPH radicals were calculated according to the following: DPPH radical-scavenging activity $(\%)=\left[\left(A_{0}-A_{1}\right) / A_{0}\right]$ $\times 100$, where $A_{0}$ is the absorbance of the control (containing all reagents except the test extracts/compounds) and $A_{1}$ is the absorbance of the extracts/compounds. The extract (or standard) concentration resulting in a $50 \%$ inhibition $\left(\mathrm{IC}_{50}\right)$ was calculated by regression equations (by plotting the extract/standard solution concentration versus percentage of inhibition). Lower IC50 values indicate higher inhibitory potential of the tested extract (16).

ABTS radical cation scavenging activity was tested according to Zou et al. (16). ABTS radical cations were produced by mixing 
equal volumes of $\mathrm{ABTS}\left(7 \mathrm{mM}\right.$ in $\mathrm{H}_{2} \mathrm{O}$ ) and potassium persulfate (4.9 $\mathrm{mM}$ in $\mathrm{H}_{2} \mathrm{O}$ ) and allowing them to react for $12-16 \mathrm{~h}$ at room temperature in the dark. The ABTS radical solution was then diluted with $96 \%$ ethanol to obtain an absorbance of about 0.7 at $734 \mathrm{~nm}$ using a spectrophotometer. $10 \mu \mathrm{L}$ of extract, or standard trolox, was added to $190 \mu \mathrm{L}$ of ABTS radical solution in a 96-well microplate. The mixture was incubated at room temperature in the dark for $30 \mathrm{~min}$. Then, absorbance readings were taken at $734 \mathrm{~nm}$. Tests were carried out in triplicate.

The percent radical scavenging activity of extracts and compounds against $A B T S$ radical were calculated according to the following: ABTS radical-scavenging activity $(\%)=\left[\left(A_{0}-A_{1}\right) / A_{0}\right]$ $\times 100$ where $A_{0}$ is the absorbance of the control (containing all reagents except the test extracts/compounds) and $A_{1}$ is the absorbance of the extracts/compounds. The extract (or standard) concentration resulting in a $50 \%$ inhibition $\left(\mathrm{IC}_{50}\right)$ was calculated by regression equations (by plotting the extract/standard solution concentration versus percentage of inhibition). Lower $I_{50}$ values indicate higher inhibitory potential of the tested extract (16).

The anti-lipoxygenase activity was evaluated as described by Phosrithong and Nuchtavorn (17) with slight modifications described by Yıldırım et al. (18). $10 \mu \mathrm{L}$ of extract or standard indomethacin were added to $20 \mu \mathrm{L}$ ethanol, $20 \mu \mathrm{L}$ pure water, $25 \mu \mathrm{L}$ of sodium borate buffer solution $(0.1 \mathrm{M}, \mathrm{pH}$ ) followed by an addition of $25 \mu \mathrm{L}$ of type $\mathrm{V}$ soybean lipoxygenase solution in buffer ( $\mathrm{pH} 9,20.000 \mathrm{U} / \mathrm{mL}$ ). The mixture was preincubated at 25 ${ }^{\circ} \mathrm{C}$ for $5 \mathrm{~min}$. Then, $100 \mu \mathrm{L}$ of $0.6 \mathrm{mM}$ linoleic acid solution was added, mixed well and the change in absorbance at $234 \mathrm{~nm}$ was recorded for $6 \mathrm{~min}$. Tests were carried out in triplicate. The inhibition percentage was calculated from the following equation: $\%$ inhibition $=\left[\left(A_{\text {control }}-A_{\text {sample }}\right) / A_{\text {control }}\right] \times 100$.

The extract (or standard) concentration resulting in a 50\% inhibition $\left(\mathrm{IC}_{50}\right)$ was calculated by regression equations (by plotting the extract/standard solution concentration versus percentage of inhibition). Lower $\mathrm{IC}_{50}$ values indicate higher inhibitory potential of the tested extract (16).

\section{Determination of the Total Phenolic and Flavonoid Con- tents}

The total phenolic content of the extract was measured as described by Gao et al. (19) with slight modifications described by Yıldırım et al. (18) Ten $\mu \mathrm{L}$ of the extract in various concentrations was mixed with $20 \mu \mathrm{L}$ of the Folin-Ciocalteu reagent, $200 \mu \mathrm{L}$ of $\mathrm{H}_{2} \mathrm{O}$, and $100 \mu \mathrm{L}$ of $15 \% \mathrm{Na}_{2} \mathrm{CO}_{3}$, and the absorbance was measured at $765 \mathrm{~nm}$ after $2 \mathrm{~h}$ of incubation at room temperature. The total phenolic content was calculated on the basis of the calibration curve of standard gallic acid and expressed as $\mathrm{mg}$ gallic acid equivalent (GAE) per $g$ dried extract.

Total flavonoid content was determined following a method by Zhang et al. (20) with slight modifications described by Yıldırım et al. (18). $25 \mu \mathrm{L}$ extract in various concentrations was mixed with $125 \mu \mathrm{L}$ of ultra-pure water and $7.5 \mu \mathrm{L}$ of $5 \% \mathrm{NaNO}_{2}$. After $6 \mathrm{~min}, 15 \mu \mathrm{L}$ of $10 \% \mathrm{AlCl}_{3} \cdot 6 \mathrm{H}_{2} \mathrm{O}$ was added. After $5 \mathrm{~min}, 50 \mu \mathrm{L}$
$\mathrm{NaOH}(1 \mathrm{M})$ was added and the solution completed with 250 $\mu \mathrm{L}$ of ultra-pure water. The absorbance was measured against the reagent blank at $510 \mathrm{~nm}$. The total flavonoid content was calculated on the basis of the calibration curve of standard quercetin and expressed as mg quercetin equivalent (QE) per $\mathrm{g}$ dried extract.

\section{Animals}

A total of 24 female Sprague-Dawley rats (250-260 g; 4-5 months) were obtained from the Animal Centre (DEHAMER, Istanbul). The animals were housed in laboratory conditions and fed ad libitum. All protocols were carried out following the project submitted to the Marmara University Animal Care and Use Committee (Protocol number: 46.2020.mar). All animal experiments complied with the guidance of the Council of International Organization of Medical Sciences (WHO/UNESCO), NIH and $\mathrm{PHS}$.

\section{Animal Experimental Procedure}

After $20 \mathrm{~h}$ of fasting, colitis was induced under anaesthesia, where an 8-cm soft cannula was inserted into the rectal hole and pushed forward. Subsequently, $5 \%$ (v/v) AA diluted in saline $(\mathrm{pH} 2.3)$ was administered in a volume of $1 \mathrm{~mL}$ (2). The control group was administered $1 \mathrm{~mL}$ saline intrarectally in lieu of AA. The rats were divided into two main groups: the control group and the AA-induced colitis group. The colitis group was further divided into three subgroups: the saline-treated (AA), the MoC extract-treated $(A A+M o C)$, and SS-treated $(A A+S S)$ groups, with each group consisting of 6 rats. Immediately after colitis induction and for the next 2 days, while saline was given to the control and AA groups, MoC extract $(300 \mathrm{mg} / \mathrm{kg}$ ) was given to the $A A+M o C$ group and SS $(100 \mathrm{mg} / \mathrm{kg})$ was given to the $A A+S S$ group. Thus, all treatments were given a total of 3 times. MoC extract and dissolved SS were administered to the rats using an oral gavage tube (p.o.) The dose of the MoC extract was selected based on a former study that demonstrated the protective effect of MoC on the Crohn's colitis model (10). However, SS, which is the standard drug for the treatment of ulcerative colitis, was used as a reference to evaluate the effectiveness of MoC (2). All treatments were given at around 11:00 am. The rats were euthanized (Thiopenthal sodium, $50 \mathrm{mg} / \mathrm{kg} / \mathrm{i} . \mathrm{p}$.) at the $72 \mathrm{nd} \mathrm{h}$ of the colitis induction. The last $8 \mathrm{~cm}$ of the distal part of the colon was harvested. Colon tissues were taken for macroscopic and histological examinations, while the remaining parts were kept at $-80^{\circ} \mathrm{C}$ until biochemical experiments were performed.

\section{IL-1 $\beta$, Malondialdehyde (MDA) and Glutathione (GSH) Lev- els and MPO Activity Measurement in Colonic Tissues}

An enzyme-linked immunosorbent assay (ELISA) test was performed to determine IL-1 $\beta$ levels in the colonic samples following the manufacturer's instructions (EBIOSCIENCE, Thermo Fisher Scientific, MA, USA). MDA levels were defined for end products of membrane lipid oxidation by capturing the generation of thiobarbituric acid-sensitive substances as previously described by Buege and Aust (21) and the data were expressed as $\mathrm{nmol} \mathrm{MDA} / \mathrm{g}$ tissue. Cellular antioxidant GSH levels were determined using a modified Ellman procedure (22) and the data 
were expressed as $\mu \mathrm{mol} \mathrm{GSH} / \mathrm{g}$ tissue. MPO activity assays were used in the evaluation of tissue neutrophil recruitment in line with Bradley et al. (23) and the data were expressed as U/g.

\section{Immunoblotting}

Frozen tissues were prepared in a $20 \mathrm{mM}$ Tris- $\mathrm{HCl}$ buffer containing protease inhibitors. Following centrifugation, the pellets were incubated in the protease inhibitor solution (which includes DTT, glycerol, Tris-HCl, EDTA, and Triton X-100) for $1 \mathrm{~h}$. After determining the amount of protein in each of the colon tissues by the Lowry method (24), samples containing $100 \mathrm{mg}$ of protein were prepared for SDS-PAGE separation. The prepared samples were loaded onto the gel and subsequently transferred to nitrocellulose membranes (at $90 \mathrm{~V}$ for $1 \mathrm{~h}$ ). The transferred samples were incubated with primary antibodies [ $\beta$-actin (sc-130657, 1: 200), bax (sc-20067, 1: 100), bcl-2 (sc7382, 1: 200), casp-3 (sc-56053, 1: 200) and casp-9 (sc-56076, 1: 200] for $14 \mathrm{~h}$, and then the membranes were incubated with rabbit monoclonal anti-goat IgG secondary antibodies (1: 1500) for an additional $1 \mathrm{~h}$. All incubations with antibodies were carried out at $+4{ }^{\circ} \mathrm{C}$. A publicly available software was used for the densitometric analysis of the resulting membranes (Bio-Rad Molecular Analyst, www.totallab.com).

\section{Morphological and Histopathological Analysis}

Macroscopic scoring of the colonic damage was performed using the criteria outlined in Table 1, with a maximum score of 10, and photographed according to Karakoyun et al. (2). The colonic tissue samples were processed for light microscopic investigations. In short, the tissues were fixed in $10 \%$ formaldehyde, dehydrated in ethanol series with ascending alcohol concentrations, cleared in xylene, and embedded in paraffin blocks. The cut tissue sections ( $5 \mu \mathrm{m}$ thick) were stained with hematoxylin and eosin (H\&E) and photographed using a light microscope with its dedicated camera (Olympus BX51; Olympus DP72; Olympus, Tokyo, Japan). Semi-quantitative histological scoring was performed according to the method modified from the previous study (2) The relevant scoring criteria are stated in Table 2.

Table 1. Criteria of the macroscopic scoring of the colonic injury.

\section{Score View}

0 Normal view

1 Local hyperaemia, no ulcers

2 Non-hyperaemic ulceration and bowel wall thickening

3 Ulceration with inflammation at one region

4 Ulceration and inflammation in two or more regions

5 Colonic damage more than $1 \mathrm{~cm}$

6-10 Damage is increased by 1 point for each $\mathrm{cm}$

(Maximum score 10)
Table 2. Criteria of the microscopic scoring of the colonic injury.

\begin{tabular}{ll}
\hline Score & Appearance \\
\hline 0 None; 1 Mild; 2 Moderate; 3 Severe & Submucosal oedema \\
\hline 0 None; 1 Localized; 2 Moderate; 3 Severe & Damage/necrosis \\
\hline 0 None; 1 Mild; 2 Moderate; 3 Severe & $\begin{array}{l}\text { Inflammatory cell } \\
\text { infiltration }\end{array}$ \\
\hline 0 None; 1 Mild; 2 Moderate; 3 Severe & Haemorrhage \\
\hline 0 Absent; 1 Present & Perforation \\
\hline (Maximum score 13) & \\
\hline
\end{tabular}

\section{Statistical Analysis}

GraphPad software (Prism 6.0; GraphPad Software, San Diego, (A, USA) was used for statistical analysis. The comparisons through all groups were made with analysis of one-way variance followed by Bonferroni multiple comparison post-hoc tests. All data were expressed as mean $\pm S D$, and $p<0.05$ was conceded to be statistically significant.

\section{RESULTS}

Extraction Yield, In Vitro Biological Activity, and Total Phenol and Flavonoid Content of MoC Extract

$22.04 \mathrm{~g}$ dried $\mathrm{MoC}$ extract was obtained from $411.71 \mathrm{~g}$ fresh fruit (extraction yield: $5.35 \%$ ). MoC extract displayed moderate DPPH radical scavenging activity with an $\mathrm{IC}_{50}$ of $222.70 \pm 12.16 \mu \mathrm{g} / \mathrm{mL}$ when compared to an $I_{50}$ value of $17.6 \pm 0.37 \mu \mathrm{g} / \mathrm{mL}$ of standard ascorbic acid $(p<0.05)$. The extract showed moderate ABTS radical scavenging activity with an $\mathrm{IC}_{50}$ value of $349.30 \pm 3.47$ $\mu \mathrm{g} / \mathrm{mL}$ when compared to standard Trolox $\left(\mathrm{IC}_{50}=13.00 \pm 0.21\right.$ $\mu \mathrm{g} / \mathrm{mL} ; \mathrm{p}<0.05)$. MoC extract with an $\mathrm{IC}_{50}$ value of $14.46 \pm 1.33$ $\mu \mathrm{g} / \mathrm{mL}$ showed potent anti-inflammatory activity compared to standard Indomethacin $\left(\mathrm{IC}_{50}=22.39 \pm 0.26 \mu \mathrm{g} / \mathrm{mL} ; \mathrm{p}<0.05\right)$. The total phenolic and total flavonoid contents of the extract were 26.16 $\pm 2.01 \mathrm{mg} \mathrm{GAE}$ and $1.88 \pm 0.02 \mathrm{mg}$ QE per $\mathrm{g}$ extract, respectively (Table 3).

\section{IL-1 $\beta$, MDA and GSH Levels, MPO Activity}

The pro-inflammatory cytokine IL-1 $\beta$ levels of the saline-treated AA group were found to be significantly higher than those of the control group $(p<0.001)$. The treatment of the rats with $\mathrm{MoC}$ and SS decreased IL-1 $\beta$ elevation $(p<0.01-p<0.001$, respectively; Figure 1a).

In comparison to the control group, while a significant elevation was observed in the MDA levels which indicate lipid peroxidation of the saline-treated AA group $(p<0.01)$, this elevation significantly decreased in the MoC and SS treated colitis groups ( $p<0.01$; Figure $1 \mathrm{~b}$ ). The cellular antioxidant molecule GSH levels were markedly diminished in the saline-treated AA group in comparison to the control group $(p<0.01)$; however, a significant elevation of GSH levels was detected in the MoC and SS-treated colitis groups $(p<0.01$ and $p<0.01$, 
Table 3. Anti-inflammatory, antioxidant activity and total compound content of MoC extract.

\begin{tabular}{|c|c|c|c|c|}
\hline Assays & MoC* & Trolox & Ascorbic acid & Indomethacin \\
\hline ABTS radical scavenging activity $\left(\mathrm{IC}_{50}, \mu \mathrm{g} / \mathrm{mL}\right)$ & $349.30 \pm 3.47^{b}$ & $13.00 \pm 0.21^{\mathrm{a}}$ & & \\
\hline DPPH radical scavenging activity $\left(\mathrm{IC}_{50}, \mu \mathrm{g} / \mathrm{mL}\right)$ & $222.70 \pm 12.16^{b}$ & & $17.6 \pm 0.37^{a}$ & \\
\hline Anti-lipoxgenase activity $\left({ }^{\prime} C_{50}, \mu g / m L\right)$ & $14.46 \pm 1.33^{\mathrm{a}}$ & & & $22.39 \pm 0.26^{b}$ \\
\hline Total phenolic content (mg GAE/g extract)** & $26.16 \pm 2.01$ & & & \\
\hline Total flavonoid content (mg QE/g extract) ${ }^{* * *}$ & $1.88 \pm 0.02$ & & & \\
\hline $\begin{array}{l}{ }^{*} \text { MoC: Ethanol extract of Momordica charantia L. fruits } \\
{ }^{* *} \text { Total phenolic content was expressed as gallic acid equival } \\
* * * \text { Total flavonoid content was expressed as quercetin equive } \\
* * * * \text { Each value in the table is represented as mean } \pm S D(n=3) \text {. }\end{array}$ & etter superscripts in & line indicat & nt differen & \\
\hline
\end{tabular}

respectively; Figure 1c). MPO activity, which indicates tissue neutrophil recruitment, was found to be significantly increased in the saline-treated AA group in comparison to the control group $(p<0.001)$. The colonic MPO activity was de-

a

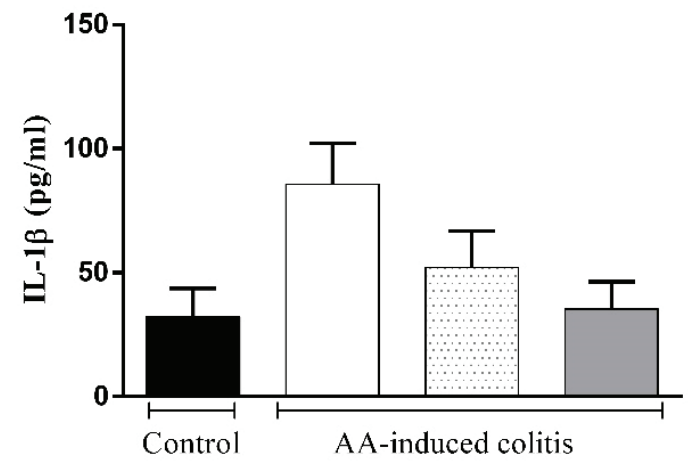

C

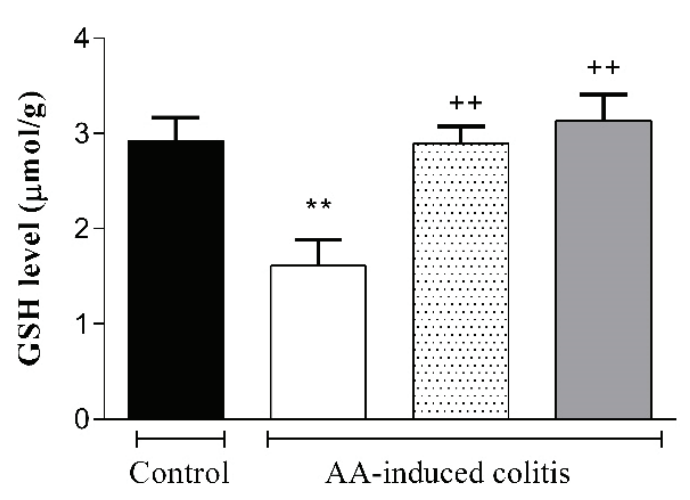

Control AA-induced colitis

C creased upon MoC and SS treatments in comparison to the saline-treated AA group ( $p<0.01$ and $p<0.001$, respectively; Figure $1 \mathrm{~d}$ ). There was no statistical difference between $\mathrm{MoC}$ and SS treatments. b

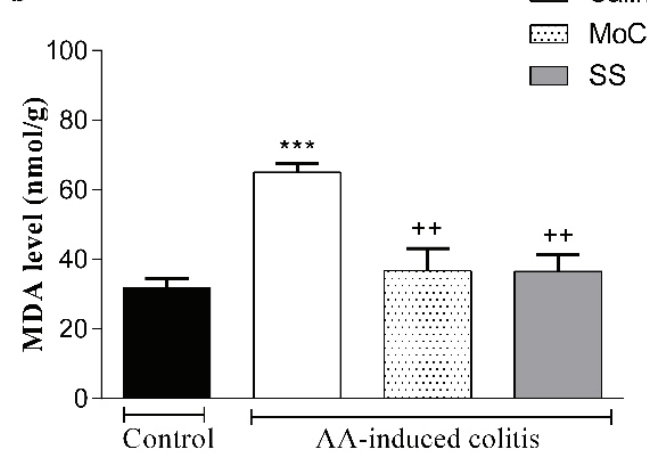

d

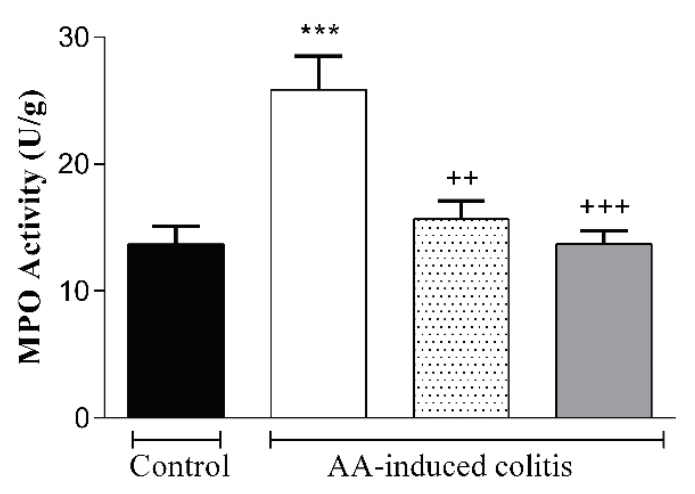

Figure 1. Tissue IL-1 $\beta$ (a), MDA (b), GSH levels (c), and MPO activity (d). ${ }^{* *} p<0.01$, and ${ }^{* * *} p<0.001$ Comparisons according to the control group, ${ }^{++} p<0.01$ and ${ }^{+++} p<0.001$ Comparisons according to the saline-treated AA-induced colitis group. Control: Saline-treated control group, AA: Saline-treated AA-induced colitis group, MoC: MoC extract-treated AA-induced colitis group, SS: SS-treated AA-induced colitis group (for each group, $\mathrm{n}=6$ ). Abbreviations: Interleukin 1- $\beta$ (IL-1 $\beta$ ); Malondialdehyde (MDA); Glutathione (GSH); Myeloperoxidase (MPO); Acetic acid (AA); Sulfasalazine (SS); Momordica charantia L. (MoC). 


\section{Immunoblotting}

The representative immunoblotting membranes of the colon tissues were illustrated in Figure 2a. Changes in the bax/bcl-2 expression ratio and casp-9, casp-3 levels were used to determine the mitochondrial apoptosis evaluation for each group $(n=4)$.

The bax/bcl-2 ratio was increased in the saline-treated AA-induced colitis group compared to the control group $(p<0.001)$. The changes in the expression of bax/bcl-2 ratio in the $A A$-induced colitis group were restored upon MoC and SS treatments $(p<0.01$ and $p<0.001$, respectively; Figure $2 b)$. Casp-9 and casp3 levels were also significantly increased in the saline-treated $A A$-induced colitis group compared to the control group $(p<0.05$ and $p<0.01$, respectively). MoC and SS treatments decreased casp- 9 and casp-3 levels compared to the saline-treated AA group ( $p<0.05$; Figure $2 c$ and $2 d$ ).

\section{Morphological and Histopathological Analysis}

At $72 \mathrm{~h}$ after colitis induction, macroscopic scoring was found to be significantly higher in the saline-treated AA group compared to the control group $(p<0.001)$, while this scoring was significantly decreased in the MoC and SS-treated colitis groups $(p<0.001$; Figures $3 a$ and $c)$.

As a result of light microscopic examinations, colon tissues of the control group had histological layers with regular morphology and the total microscopic damage score was the lowest (mean 1.46; Figure 3b). In the saline-treated AA group, severe loss of epithelial lining, extensive submucosal oedema, massive inflammatory cell infiltration, and haemorrhage were observed with the highest total damage score (mean 12.4). MoC and SS-treated colitis groups showed similar histological appearance, such as more regular epithelial surfaces, decreased inflammatory cell infiltration, and submucosal oedema (Figure 4). Microscopic scoring was found to be markedly higher in the AA group compared to the control group $(p<0.001)$, while this scoring was significantly decreased upon MoC and SS treatment $(p<0.001)$. When compared to the control group, there were still high scores in the MoC and SS groups $(p<0.01$ and $p<0.001$, respectively), whereas the difference between these treatment groups was not significant (Figure 3b, Table 4). a
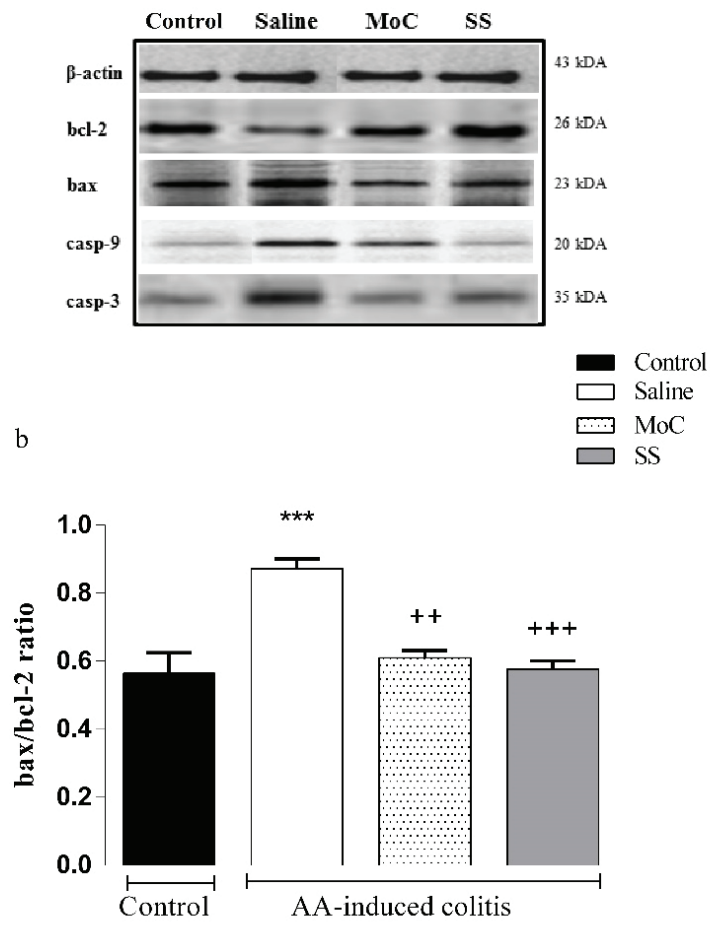

c

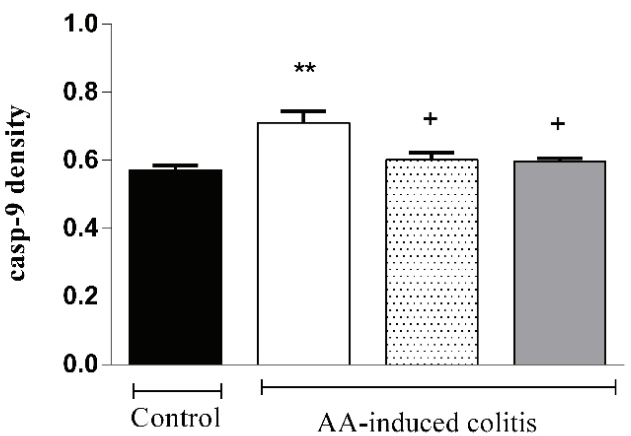

d

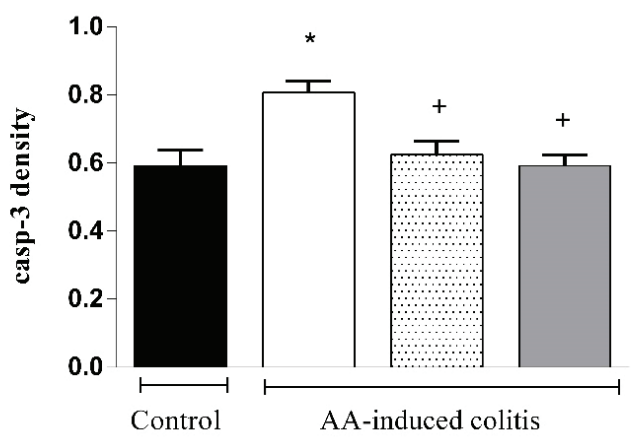

Figure 2. The representative images of membranes obtained from western blotting assays showing protein expressions of $\beta$-actin, bax, bcl 2, casp-9, and casp-3 (a), the expression levels of bax/bcl-2 ratio (b), casp-9 (c) and casp-3 (d) in colon.

All membranes (for each group, $n=4$ ) were normalized by using $\beta$-actin antibodies . ${ }^{*} p<0.05,{ }^{* *} p<0.01$, and ${ }^{* * *} p<0.001$ Comparisons according to control group, ${ }^{+} p<0.05,{ }^{++} p<0.01$ and ${ }^{++} p<0.001$ Comparisons according to saline treated AA-induced colitis group. Control: Saline-treated control group, AA: Saline-treated AA-induced colitis group, MoC: Momordica charantia extract-treated AA-induced colitis group, SS: SS-treated AA-induced colitis group (for each group, $\mathrm{n}=6$ ). Abbreviations: $\mathrm{Bcl}$-2-associated X protein (bax); $\mathrm{B}$-cell lymphoma 2 (bcl-2); Caspase-9 (casp-9); Caspase-3 (casp-3); Acetic acid (AA); Sulfasalazine (SS); Momordica charantia L. (MoC). 
a

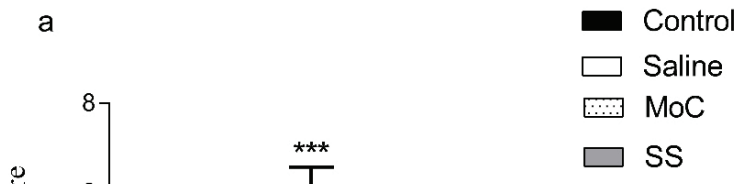

b

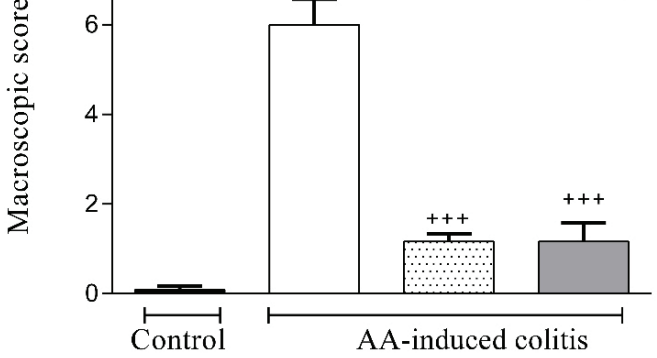

C
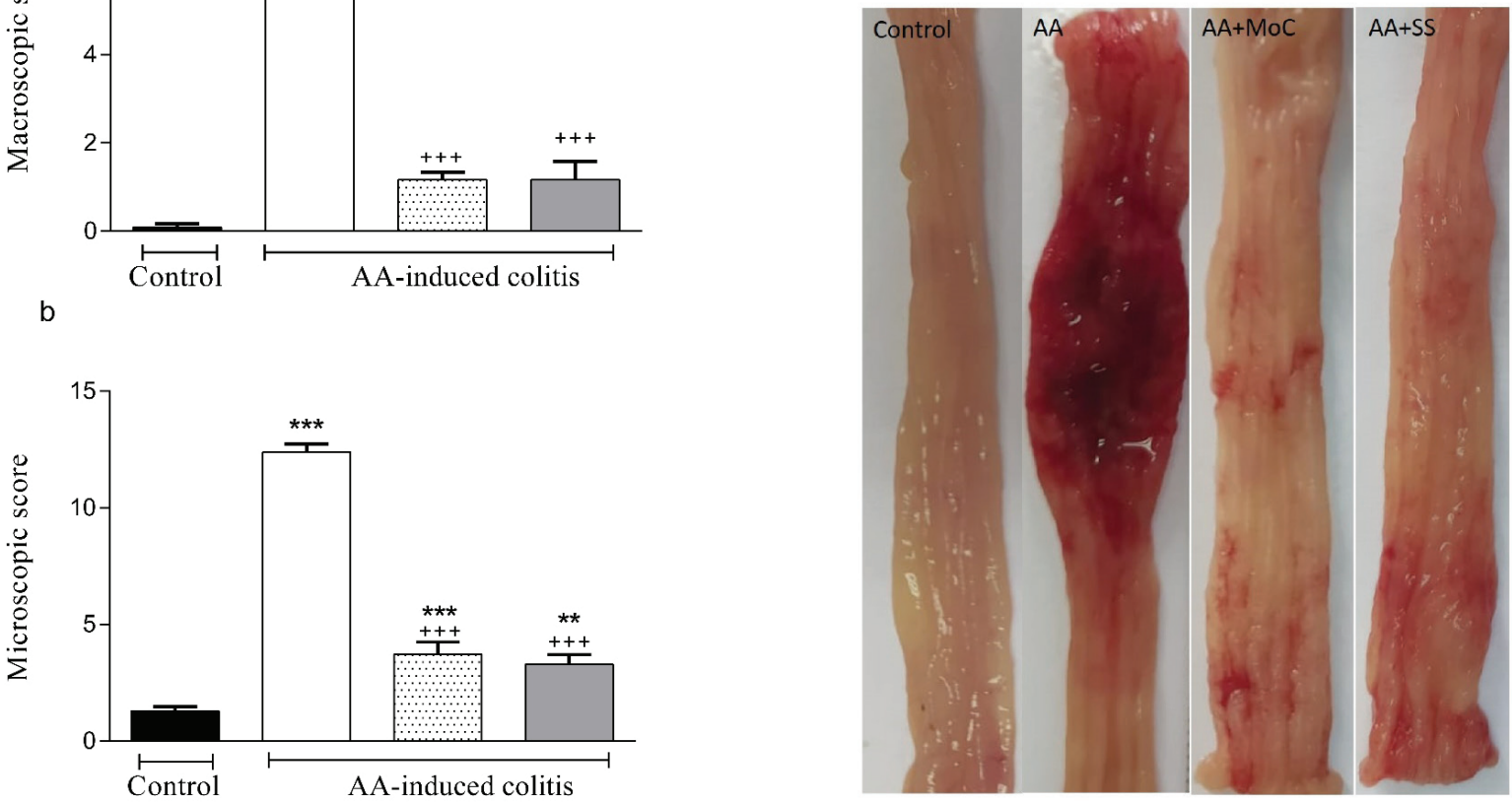

Figure 3. Macroscopic (a), microscopic scoring (b), and representative photographs of the colonic tissues (c). ${ }^{* *} p<0.01$, and ${ }^{* * *} p<0.001$ Comparisons according to control group ${ }^{+++} \mathrm{p}<0.001$ Comparisons according to saline-treated AA-induced colitis group. Control: Salinetreated control group, AA: Saline-treated AA-induced colitis group, MoC: MoC extract-treated AA-induced colitis group, SS: SS-treated AA-induced colitis group (for each group, $n=6$ ). Abbreviations: Acetic acid (AA); Sulfasalazine (SS); Momordica charantia L. (MoC).

Table 4. Predominant histological grading of each sign of colonic damage in each experimental group of animals.

\begin{tabular}{lcccccc}
\hline & Oedema & Damage/Necrosis & Inflammatory cell & Haemorrhage & Perforation & Total Score \\
\hline Control & 0.38 & 0.42 & 0.44 & 0.02 & 0.20 & 1.46 \\
\hline Saline+AA & 3.00 & 2.70 & 3.00 & 2.90 & 0.80 & 12.40 \\
\hline MoC+AA & 0.90 & 0.86 & 1.22 & 0.56 & 0.20 & 3.74 \\
\hline SS+AA & 0.80 & 0.65 & 1.125 & 0.50 & 0.25 & 3.30 \\
\hline Abbreviations: Acetic acid (AA); Sulfasalazine (SS); Momordica charantia L. (MoC). & & &
\end{tabular}

\section{DISCUSSION}

The results of the current study reveal that $\mathrm{MoC}$ extract ameliorates colonic damage, as assessed by decreased pro-inflammatory cytokine IL-1 $\beta$ levels, bax/bcl-2 ratios, caspase-3 and -9 levels, MPO activity, lipid peroxidation and increased GSH levels. Also, macroscopic and histopathologic evaluation suggests significant colonic healing.

In the present study in vitro DPPH and ABTS radical scavenging activity and lipoxygenase inhibitory activity, and in vivo MPO enzyme activity of MoC extract were investigated. The antiox- idant and anti-lipoxygenic effects of MoC extract detected in vitro confirmed its in vivo antioxidant and anti-inflammatory activity. Previous studies have shown that MoC contained cucurbitane-type triterpenes as the main compounds (8, 9, 25-27). Furthermore, some of these triterpene compounds have been reported to have significant antioxidant and anti-inflammatory activities $(27,28)$. Therefore, these cucurbitane-type triterpenes together with other compounds in $\mathrm{MoC}$ may be responsible for the antioxidant and anti-inflammatory activity of MoC. In our in vitro analysis MoC exhibited a strong anti-inflammatory activity compared to standard Indomethacin, which is an important 
Eur J Biol 2021; 80(2): 119-128

Ozbeyli et al. Momordica charantia L. Heals Ulcerative Colitis
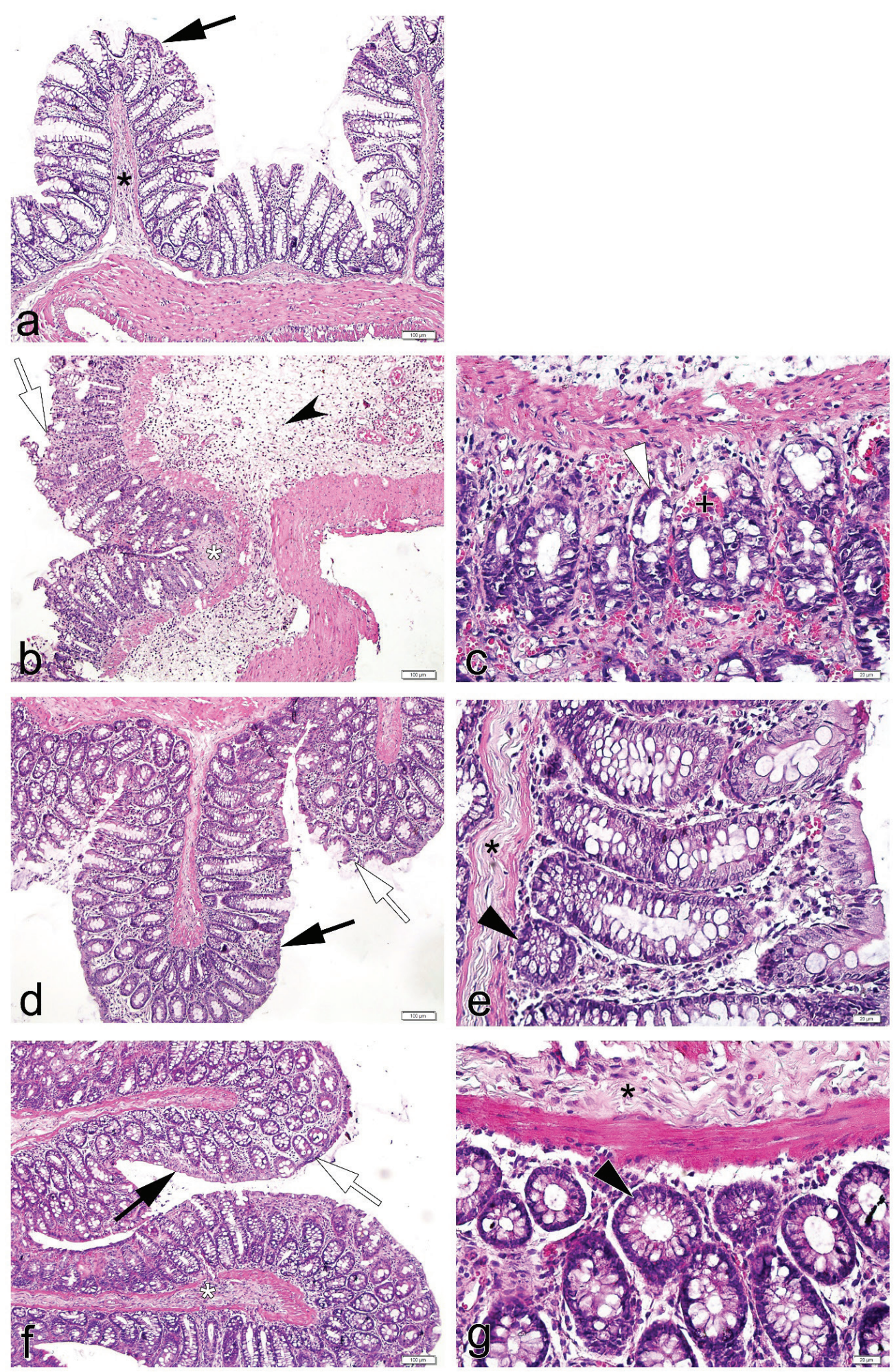

Figure 4. Representative light microscopic photographs of the colonic tissues from all experimental groups. (a) Saline-treated control group, (b-c) Saline-treated AA-induced colitis group (AA), (d-e) MoC extract-treated AA-induced colitis group (AA+MoC) group. (f-g) SS-treated AA-induced colitis group (AA+SS) group. Arrow: Normal colon mucosa with regular surface epithelium. Arrowhead: Regular Lieberkuhn crypts. Asterisk $(*)$ : Regular submucosa. Notched arrow: Inflammatory cell infiltration. Plus (+): Vascular dilatation/haemorrhage. White arrow: Colon mucosa with damaged epithelium. White arrowhead: Lieberkuhn crypts with the abnormal organization. H\&E staining. Bar size a,b,d,f: $100 \mu \mathrm{m}$ and c,e,g: $20 \mu \mathrm{m}$. Abbreviations: Acetic acid (AA); Sulfasalazine (SS); Momordica charantia L. (MoC). 
reason for colonic healing. However, in the present study, the total phenolic and flavonoid contents of $\mathrm{MoC}$ were found to be low. The fact that MoC is rich in cucurbitane-type triterpene compounds overlaps with this result.

Here, we established an AA-induced colitis model because it is highly similar to human inflammatory bowel disease (IBD) in terms of pathophysiological, histopathologic, and inflammatory mediator profiles (29). AA-induced UC has been shown to initiate physical and chemical damage in colon tissue, causing inflammation and an increase in ROS levels and a subsequent decrease in GSH levels. Besides, neutrophils that infiltrate the damaged colon mucosa, which leads to oxidative stress, also increase inflammation $(4,30,31)$. In the present study, impaired epithelial integrity, which was demonstrated macroscopically and microscopically, facilitated neutrophil infiltration from blood to colon tissue. Our in vitro biological activity results indicate that $\mathrm{MoC}$ extract has free radical scavenging and anti-inflammatory activities. Scavenging of free radicals and depletion of inflammation diminishes lipid peroxidation and restores cellular GSH stores. Decreased MDA levels and increased GSH levels observed upon MoC treatment in this study may be due to such activities of MoC. In addition, MoC therapy reduced MPO activity, which increased oxidative damage, and inflammation caused by AA increased neutrophil infiltration into the colon, mucosal and submucosal necrosis, oedema, vascular dilation, immense epithelial damage, and submucosal ulceration, which are main features of human colitis (29). Our macroscopic scoring showed that AA caused excessive damage to the colon tissue, but the MoC and SS treatments had significant ameliorative effects. Our macroscopic and microscopic results were in agreement with our biochemical results. Compared to the control group, there was still microscopic damage in the MoC and SS-treatment groups; however, these treatment groups were similar and the results correlated with macroscopic scoring. IL-1 $\beta$ proinflammatory cytokine is released from the colonic macrophage early in inflammation after AA administration and exacerbates mucosal inflammation $(32,33)$ and directly proportional to the severity of inflammation (34). Functional foods are beneficial by reducing pro-inflammatory cytokine expression in models of IBD (35). MoC reduced IL-1 $\beta$ levels in DSS-induced colitis and TNBS-induced colitis models $(10,15,34)$.

In line with these studies, our result showed that colitis caused a significant increase in colonic IL-1 $\beta$ levels and that MoC and SS treatments were effective in reversing this trend. However, in those studies, the preparation methods of the plant sample, the treatment durations, treatment ways, and the chemical agents used for model induction were different from our study.

Colitis has previously been shown to cause increased apoptosis in colonic epithelial cells (36-38). Overproduction of ROS in cells can disrupt cellular parts, such as lipids and proteins, which can eventually cause cell death by apoptosis (39). Anti-apoptotic protein bcl-2 is localized on the mitochondrial membrane and inhibits apoptosis by stabilizing this membrane (40). However, bax inhibits the anti-apoptotic function of bcl-2 (41). Zhu et al. demonstrated the increased expression of bax/bcl-2, casp-3, and casp-9 in a UC rat model (38). Consistent with this report, our data showed that AA increased bax/bcl-2 ratio, casp-3, and casp-9 levels in the colon. In previous studies, MoC has been reported to reduce pro-apoptotic protein casp-3 and casp-9 levels of uterine tissue in ovariectomized rats (42). The results presented herein revealed that $\mathrm{MoC}$ extract attenuated $\mathrm{AA}$-induced colonic cell apoptosis by decreasing casp- 9 and casp-3 levels and bax/bcl-2 ratio, thereby improving the epithelial barrier integrity and colonic architecture.

\section{CONCLUSION}

Our in vivo and in vitro examinations proved that $\mathrm{MoC}$ extract had a protective role in AA-induced inflammation, apoptosis, and oxidative damage. $\mathrm{MoC}$ reduced the severity of colitis. Our results point out that $\mathrm{MoC}$ extract treatment appears to be as effective as SS treatment. According to our study results, we suggest that $\mathrm{MoC}$ has a protective role in AA-induced colitis.

Acknowledgement: We thank Deniz Mukaddes Turet for taking pictures of the colon and Seren Ede for helping arrange the references.

Ethics Committee Approval: This study was approved by the Marmara University Animal Experiments Local Ethics Committee (14.09.2020 / 45.2020.mar).

Informed Consent: Written consent was obtained from the participants.

Peer Review: Externally peer-reviewed.

Author contributions: Conception/Design of study- D.O., G.S.; Data Acquisition- D.O., A.S.; Data Analysis/Interpretation- D.O., A.S., A.A., K.T., O.T.C.K., I.S.; Drafting Manuscript- D.O., A.S., A.A., O.T.C.K.; Critical Revision of Manuscript- K.T, G.S.; All the authors reviewed the manuscript and after their respective inputs gave their final approval for submission/publication.

Conflict of Interest: Authors declared no conflict of interest.

Financial Disclosure: Authors declared no financial support.

\section{REFERENCES}

1. Yadav V, Varum F, Bravo R, Furrer E, Bojic D, Basit AW. Inflammatory bowel disease: exploring gut pathophysiology for novel therapeutic targets. Transl Res 2016; 176: 38-68.

2. Karakoyun B, Ertaş B, Yüksel M, Akakin D, Çevik Ö, Şener G. Ameliorative effects of riboflavin on acetic acid-induced colonic injury in rats. Clin Exp Pharmacol Physiol 2018; 45: 563-72.

3. Fabia R, Willen R, Arrajab A, Andersson R, Ahren B, Bengmark S. Acetic acid-induced colitis in the rat: a reproducible experimental model for acute ulcerative colitis. Eur Surg Res 1992; 24: 211-25.

4. El-Akabawy G, El-Sherif NM. Zeaxanthin exerts protective effects on acetic acid-induced colitis in rats via modulation of pro-inflammatory cytokines and oxidative stress. Biomed Pharmacother 2019; 111: 841-51.

5. Shalkami AS, Hassan MIA, Bakr AG. Anti-inflammatory, antioxidant and anti-apoptotic activity of diosmin in acetic acid-induced ulcerative colitis. Hum Exp Toxicol 2018; 37: 78-86. 
6. Hagiwara C, Tanaka $\mathrm{M}$, Kudo $\mathrm{H}$. Increase in colorectal epithelial apoptotic cells in patients with ulcerative colitis ultimately requiring surgery. J Gastroenterol Hepatol 2002; 17: 758-64.

7. Rosenberg LN, Peppercorn MA. Efficacy and safety of drugs for ulcerative colitis. Expert Opin Drug Saf 2010; 9: 573-92.

8. Saeed F, Afzaal M, Niaz B, Arshad Mu, Tufail T, Hussain MB, et al. Bitter Melon (Momordica Charantia): A Natural Healthy Vegetable. Int J Food Prop 2018; 21: 1270-90.

9. Huang $\mathrm{Ht}$, Zhang LJ, Huang $\mathrm{HC}$, Hwang SY, Wu Cl, Lin YC, et al. Cucurbitane-type triterpenoids from the vines of Momordica charantia and their anti-inflammatory activities. J Nat Prod 2020; 83: 1400-08.

10. Ünal NG, Kozak A, Karakaya S, Oruç N, Barutçuoğlu B, Aktan Ç, et al. Anti-inflammatory effect of crude Momordica charantia I. extract on 2,4,6-trinitrobenzene sulfonic acid-induced colitis model in rat and the bioaccessibility of its carotenoid content. J Med Food 2020; 23: 641-8.

11. Patel S, Patel T, Parmar K, Bhatt Y. Patel NM. Isolation, characterization and antimicrobial activity of charantin from Momordica charantia linn. fruit. Int J Drug Dev Res 2010; 2: 629-34.

12. Kumar R, Balaji S, Sripriya R, Nithya N, Uma TS, Sehgal PK. In vitro evaluation of antioxidants of fruit extract of Momordica charantia I. On fibroblasts and keratinocytes. J Agric Food Chem 2010; 58: 1518-22.

13. Jia S, Shen M, Zhang F, Xie J. Recent advances in Momordica charantia: functional components and biological activities. Int J Mol Sci 2017; 18: 2555.

14. Gürbüz I, Akyüz Ç, Yeşilada E, Şener B. Anti-ulcerogenic effect of Momordica charantia I. fruits on various ulcer models in rats. J Ethnopharmacol 2000; 71: 77-82.

15. Lu HY, Lin BF. Wild bitter melon alleviates dextran sulphate sodium-induced murine colitis by suppressing inflammatory responses and enhancing intestinal regulatory t cells. J Funct Foods 2016; 23: 590-600.

16. Zou Y, Chang SK, Gu Y, Qian SY. Antioxidant activity and phenolic compositions of lentil (Lens culinaris var. morton) extract and its fractions. J Agric Food Chem 2011; 59: 2268-76.

17. Phosrithong N, Nuchtavorn N. Antioxidant and anti-inflammatory activities of Clerodendrum leaf extracts collected in Thailand. Eur J Integr Med 2016; 8(3): 281-85.

18. Yildirim A, Şen A, Dogan A. Bitis L. Antioxidant and anti-inflammatory activity of capitula, leaf and stem extracts of tanacetum cilicicum (boiss.) Grierson. Int J Second Metab 2019; 6: 211-22.

19. Gao, X, Ohlander M, Jeppsson N, Björk L, Trajkovski V. Changes in Antioxidant Effects and Their Relationship to Phytonutrients in Fruits of Sea Buckthorn (Hippophae rhamnoides L.) during Maturation. J Agric Food Chem 2000; 48(5): 1485-90.

20. Zhang R, Zeng Q, Deng Y, Zhang M, Wei Z, Zhang Y, et al. Phenolic profiles and antioxidant activity of litchipulp of different cultivars cultivated in Southern China. Food Chem. 2013; 136: 169-76.

21. Buege JA, Aust SD. Microsomal lipid peroxidation. Meth Enzymol 1978; 52: 302-10.

22. Beutler E. Red Cell Metabolism. A manual of biochemical methods. In B ergmeyen HV (ed.), annals of internal medicine 1975 2nd Ed. Issue 6. Grune \& Stratton.

23. Bradley PP, Priebat DA, Christensen RD, Rothstein G. Measurement of cutaneous inflammation: estimation of neutrophil content with an enzyme marker. J Investig Dermatol 1982; 78: 206-9.

24. Lowry OH, Rosebrough NJ, Farr AL, Randall RJ. Protein measurement with the folin phenol reagent. J Biol Chem 1951;193: 265-75.
25. Wurtz NR, Viet A, Shaw SA, Dilger A, Valente MN, Khan JA, et al. Potent triazolopyridine myeloperoxidase inhibitors. Acs Med Chem Lett 2018; 9: 1175-80.

26. Chang $\mathrm{Cl}$, Chen CR, Liao YW, Cheng HI, Chen YC, Chou CH. Cucurbitane-type triterpenoids from Momordica charantia. J Nat Prod 2006; 69: 1168-71.

27. Lin Kw, Yang Sc, Lin Cn. Antioxidant constituents from the stems and fruits of Momordica charantia. Food Chem 2011; 127: 609-14.

28. Shivanagoudra SR, Perera WH, Perez JL, Athrey G, Sun Y, Jayaprakasha GK, et al. Cucurbitane-type compounds from Momordica charantia: Isolation, in vitro antidiabetic, anti-inflammatory activities and in silico modeling approaches. Bioorg Chem 2019; 87: 31-42.

29. Randhawa PK, Singh K, Singh N, Jaggi AS. 2014. A review on chemical-induced inflammatory bowel disease models in rodents. Korean J Physiol Pharmacol 2014; 18: 279-88.

30. Iseri SO, Ersoy Y, Ercan F, Yuksel M, Atukeren P, Gumustas K, et al. The effect of sildenafil, a phosphodiesterase- 5 inhibitor, on acetic acid-induced colonic inflammation in the rat. J Gastroenterol Hepatol (Australia) 2009; 24: 1142-48.

31. Kolgazi M, Uslu U, Yuksel M, Velioglu-Ogunc A, Ercan F, Alican I. The role of cholinergic anti-inflammatory pathway in acetic acid-induced colonic inflammation in the rat. Chem Biol Interact 2013; 205(1): 72-80.

32. Soliman NA, Keshk WA, Rizk FH, Ibrahim MA. The possible ameliorative effect of simvastatin versus sulfasalazine on acetic acid induced ulcerative colitis in adult rats. Chem Biol Interact 2019; 298: 57-65.

33. Tahan G, Gramignoli R, Marongiu F, Aktolga S, Cetinkaya A, Tahan $V$, et al. Melatonin expresses powerful anti-inflammatory and antioxidant activities resulting in complete improvement of acetic-acid-induced colitis in rats. Dig Dis Sci 2011; 56: 715-20.

34. Semiz A, Ozgun Acar O, Cetin H, Semiz G, Sen, A. Suppression of inflammatory cytokines expression with Bitter melon (Momordica charantia) in tnbs-instigated ulcerative colitis. J Transl Int Med 2020; 8: 177-87.

35. Hossen I, Hua W, Ting L, Mehmood A, Jingyi S, Duoxia X, et al. Phytochemicals and inflammatory bowel disease: A review. Critical Reviews in Food Science and Nutrition 2020; 60(8): 1321-45.

36. Guven B, Can M, Piskin O, Aydin BG, Karakaya K, Elmas O, et al. Flavonoids protect colon against radiation induced colitis. Regul Toxicol Pharmacol 2019; 104: 128-32.

37. Yao J, Cao X, Zhang R, Li YX, Xu Zl, Zhang DG, et al. Protective effect of baicalin against experimental colitis via suppression of oxidant stress and apoptosis. Pharmacogn Mag 2016; 12: 225-34.

38. Zhu L, Dai LM, Shen H, Gu PQ, Zheng K, Liu YJ, et al. Qing Chang Hua Shi granule ameliorate inflammation in experimental rats and cell model of ulcerative colitis through Mek/Erk signaling pathway. Biomed Pharmacother 2019; 116: 108967.

39. Kannan K, Jain SK. Oxidative stress and apoptosis. Pathophysiology 2000; 7: 153-63.

40. Haeberlein SLB. Mitochondrial function in apoptotic neuronal cell death. Neurochem Res 2004; 29: 521-30.

41. Tan KO, Fu NY, Sukumaran SK, Chan SL, Kang JH, Poon KL, et. Map1 is a mitochondrial effector of bax. Proc Natl Acad Sci USA. 2005; 102(41): 14623-8.

42. Cevik O, Akpinar H, Oba R, Cilingir OT, Ozdemir ZN, Cetinel S, et al. The effect of Momordica charantia intake on the estrogen receptors $E S R a / E S R \beta$ gene levels and apoptosis on uterine tissue in ovariectomy rats. Mol Biol Rep 2015; 42: 167-77. 